\section{Ökologischer Konsum - Ansätze heterogener Bündnisse}

Das IÖW führte Anfang Nov.1988 seine Jahrestagung zum Thema „Ökologischer Konsum“ durch. Es setzte damit die Reihe von Tagungen fort, auf denen Felder ökologischer Politik vorgestellt und für die IÖWIVÖWProjektarbeit erschlossen werden sollten. Mit dieser Abfolge war der Anspruch verbunden, die inneren $\mathrm{Zu}$ sammenhänge zwischen diesen Themenfeldern im Auge zu behalten, also z.B. zwischen Wirtschaftsethik, ökologischer Produktion und ökologischem Konsum auch strategisch zu diskutieren. Ein kontroverser theoretischer Anknüpfungspunkt bildete die Darstellung der Konsumenteninteressen als „Kollektivgüter“. Dabei wurde die rationale Kalkulation des Individuums folgendermaßen (von Helmut Wiesenthal) beschrieben:

„Es wird eine große Zahl von individuellen Beiträgen benötigt, deren Zusammenfließen ungewiß ist, weil im Erfolgsfall jeder, Beitragende wie Abseitsstehende, Nutzen zögen. Jeder rational Beitragswillige muß damit rechnen, daß andere anders entscheiden und sich enthalten. Gleichzeitig weiß er, daß die Wirkung des eigenen Beitrags zu klein ist, um über den Erfolg zu entscheiden. Er ist entbehrlich, wenn genügend andere kooperationswillig wirken. Da alle potentiell $\mathrm{Be}$ teiligten in rationalen Überlegungen $\mathrm{zu}$ dem Schluß gelangen, ihr Beitrag sei, ohne daß sie es ändern könnten, entweder vergeblich oder überflüssig, ist das Ergebnis , rationale Passivität“...

Die Logik des isolierten Beispiels ist (also) keine brauchbare Antwort auf das Dilemma des kollektiven Handelns. Erst wenn ,passende“ Rahmen-

bedingungen hergestellt sind, machen individuelle Initiativen kollektiven Sinn. Aber schon die Gründung durchsetzungsstarker Konsumentenorganisationen wird durch das Kollektivgutproblem be- oder gar verhindert." Als Ausweg bot Wiesenthal an, ,die Suche nach homogenen und gleichgerichteten Beteiligungsmotiven aufzugeben. Stattdessen wäre offensiv ein Muster ,pluraler" Organisation anzustreben. Nicht die Gleichartigkeit von Handlungsmotiven und -bereitschaften, sondern deren optimale Verschiedenheit ermöglichen den Start einer Bewegungsdynamik und die Stabilisierung des Kooperationszusammenhangs. Statt rationales Kollektivhandeln als Resultante der gleichen Deutung identischer Situationen zu betrachten (wie etwa im Fortsetzung Seite 11

Basis: Bevölkerung 14-64 Jahre $=39,4 \mathrm{Mio}$

Typologie des allgemeinen Verbraucherverhaltens

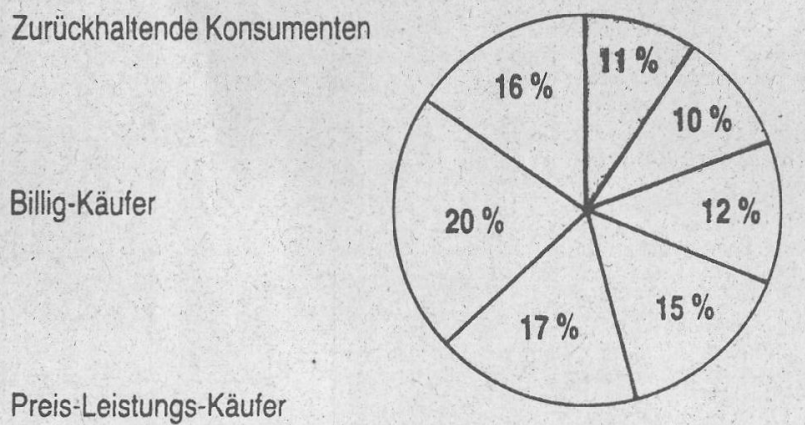

Generell Konsumfreudige

Großzügige Prestige-Käufer

Verhinderte

Viel-Konsumenten

"Bio"- und umweltbewußte Konsumenten
Neues IÖW-Gutachten im Auftrag der Fraktion Die Grünen im Bundestag.

\section{"Neuorientierung}

der bundesdeutschen Währungs-

und Finanzpolitik im

internationalen Rahmen

Der Zusammenbruch des Systems von Bretton Woods markiert nicht nur einen Knotenpunkt der Relativierung der us-amerikanischen Hegemonie, sondern auch den Beginn der Deregulierung und Globalisierung der internationalen Finanzmärkte, deren Tempo sich bis zuletzt eher weiter beschleunigt hat. Entgegen den damaligen Erwartungen in Politik und Wissenschaft hat die Flexibilisierung der Wechselkurse bei unreguliertem Kapitalverkehr zur Destabilisierung der Weltwirtschaft geführt. Der sich herausbildende Multiwährungsstandard mit dem Dollar als nach wie vor erstem Weltgeld und der DM und dem Yen als weiteren Reservewährungen intensivierte die Wührungskonkurrenz, führte zu heftigen Portfolioumschichtungen, riesigen Leistungsbilanzungleichgewichten, Überschuldung ganzer Weltregionen etc. Wirtschaftspolitische Kooperation kam nur punktuell zustande und scheiterte wiederholt an privaten internationalen Kapitalbewegungen. Vor allem die Bundesrepublik wurde zu einem Störfaktor, da sie mit ihrer handelsmerkantilistischen Strategie der Exportforcierung über stabile Preise und hohe Produktivitätsfortschritte $z u$ einem entscheidenden Blockadefaktor in der Weltwirtschaft wurde. Vor diesem Hintergrund erscheint uns eine alternative Außenwirtschaftspolitik - in der Eingrenzung auf die Konzeption von alternativer Wäh rungs-und Finanzpolitik - realistisch, in der auf eine Weiterentwicklung des Europäischen Währungssystems (EWS) orientiert wird. Dieses sollte intern demokratisch strukturiert sein und sich extern vom internationalen Kapitalmark abkoppeln. Eine Wiederbelebung weltwirtschaftlicher Insitutionalisierungen in der Form eines Weltwährungssystems halten wir angesichts der Interessengegensätze für illusorisch. Auch eine isolierte bundesrepublikanische Lösung wäre angesichts der erreichten Integration innerhalb der EG insgesamt kontraproduktiv.

Fortsetzung Seite 
Dieser Typ zeichne sich - zunächst in allg. Form charakterisiert - aus ,durch die Gleichrangigkeit der Gesprächspartner Ethik und Wirtschaftswissenschaft" (S. 28). Es handele sich bei den Forschungsansätzen dieses Typs „um die diversen ökonomischen Theorien, um die ökonomische Theorie des Rechts, die ökonomische Theorie der Verfassung, die ökonomische Theorie der Demokratie, dié ökonomische Theorie moralischer Institutionen allgemein. In Bezug auf unsere Problematik wäre das Analogon in der Tendenz nichts geringeres als eine ökonomische Theorie der Moral" (ebd.).

Was dies forschungsprogrammatisch bedeuten kann, versucht Homann in Aufnahme und Überbietung v.a. des institutional choice-Ansatzes (J.M. Buchanan) zu explizieren (vgl. ausführlicher sein „Rationalität und Demokratie“, Tübingen 1988), und es dürfte interessant sein, diese frei von Gruppenakzeptanz entwickelten Überlegungen und Ansprüche (hinsichtlich der inaugurierten ökonomischen Begründung der Moral) näher daraufhin zu belesen, ob die beanspruchte unhintergehbare Legitimität der Etablierung der modernen Einzelwissenschaften unter der Hand nicht doch wieder zu einem ökonomischen Imperialismus gegenüber der Geltung ethischer Imperative denaturiert?

Die gemeinsame Erklärung ist sich der Schwierigkeiten, ja Gefahren dieses ,anspruchsvollen Programms" zumindest insofern bewußt, dẩ nun nicht etwa die traditionalen Relationen zwischen einer führenden Ethik und einer dienenden Ökonomik gänzlich umgekehrt werden sollten und folglich , die wirtschaftswissenschaftliche Kalkulation zur ausschließlichen oder auch nur zur fundamentalen Betrachtungsweise in der Begründung moralischer Normen erkärt wird" (ebd.) . Es könne sich in der Forschung der nächsten Jahre nur um die Aufgabe handeln, ,, in der wirtschaftswissenschaftlichen Kalkulation einen Beitrag zur Begründung von Normen zu sehen und anderen Betrachtungsweisen - technischen, juristischen, psychologischen, theologischen etc. - ihr selbstverständliches Recht zu lassen" (ebd.) - zumal nicht sicher sei, ob mit diesen Ansätzen eine theoretisch befriedigende Lösung der Probleme erreichbar sei, die den Utilitarismus (als immer noch geltende theoretische Basis der Mikrofundierung der modern economics) in vorliegenden Varianten (incl. Regelutilitarismus) ,in die Krise gebracht haben: Ad hoc-Inanspruchnahmen von Ausnahmen, Menschenrechte, Minderheitenschutz etc." ( S.29).

$\mathrm{Ob}$ allerdings das ebenfalls bekundete Problembewußtsein zum wissenschaftsmethodischen und forschungspragmatischen Status der allzu lange sorglos benutzten Kunstfigur des ,homo oeconomicus" ( bzw. seiner Fortentwicklung „REMM“ $=$ resourceful evaluating maximising man) tatsächlich zu den von der Vereinsgruppe erstrebten theoretischen Fortschritten der „wirtschaftswissenschaftliche Modelle" (die nicht als Beschreibung der Realität, sondern nur als Organisation des Nachdenkens über die Realität Geltung beanspruchen) in ethischer Hinsicht führen wird, kann in Verfolgung der von Buchanan reformulierten Hobbeschen Traditionslinie zwar füglich bezweifelt, aber kaum abschließend beurteilt und als gewichtige Fortentwicklung ökonomieinterner Argumentationsmuster schon gar nicht hintergangen werden.

Neben der deshalb zu führenden Auseinandersetzung mit ambitionierten Ansätzen dieser Richtung (a la Homann) gilt es daher, einerseits (teil-)konkurrierende Analysen an derartig präfigurierte Diskurse heranzuführen, um ihre immanenten Probleme deutlicher zu fassen (vgl. bspw. die in A. Sens Royer Lectures, enthaltenen theorieimmanenten Problematisierungen der „ökonomischen Rationalität" ); andererseits aber vornehmlich auch, konkurrierende Bestimmungen des grundlegenden Verhältnisses von Ethik und Ökonomik (bspw. MacIntyre, Picht, MeierAbich -s. hierzu auch IÖW-Schriftenreihe 9/87) in diesem wesentl. nunmehr von Ökonomen getragenen wirtschaftsethischen. Diskurs zur Geltung zu bringen - insbesondere auch wegen der nicht zu übersehenden Tatsache, daß darin noch immer eine nicht mehr zeit- und problemgemäße Verengung der Wirtschaftsethik auf die gesellschaftlichen und sozialen Aspekte vorherrscht und somit die ökologischen Probleme explizit noch kaum als ,wirtschaftsethische“ Kandidaten erster Priorität wahrgenommen und thematisiert werden. Dieses bezeichnende Defizit betrifft freilich nicht nur die vorliegenden Berichtsbände der Vereinsgruppe; selbst in dem jüngst erschienenen Buch von Peter Koslowski „Prinzipien der ethischen Ökonomie“ (Tübingen 1988), welches mit dem Untertitel-Anspruch aufwartet

"Grundlegung der Wirtschaftsethik und der auf die Ökonomie bezogenen Ethik", werden nur drei knappe der insgesamt rd. 300-seitigen Ausführungen der „Natur" gewidmet.

(Entgegen dieser insofern eher konventionellen Wirtschaftsethik-Diskussion in den Vereins-Bänden sei hier - in lauterer Eigenwerbung - bereits auf den Anfang 1989 im Haupt Verlag Bern erscheinenden Band: ,Wirtschaftsethik und ökologische Wirtschaftsforschung" hingewiesen, der die überarbeiteten Beiträge zur gleichnamigen IÖW-Konferenz vom Dez. 1987 enthält)

Ob es allerdings im Rahmen der Vereinsaktivitäten künftig überhaupt eine Fortsetzung des wirtschaftsethischen Diskurses geben wird, ist bislang offiziell ungeklärt; einige darauf zielende weitergehende Formulierungen waren bereits in der publizierten Endfassung des Arbeitsberichtes gestrichen und on-dits zufolge ist selbst damit zu rechnen, daß es der Vorstand bei dieser zeitweiligen Befassung der wirtschaftsethischen Frage durch seinen „temporären Ausschuß“ bewenden lassen könnte. Dies wäre angesichts der international wachsenden Beschäftigung mit Wirtschaftsethik (wie der Gründung bspw. der European Association for Business Ethics) fraglos eine bedauerliche Abkopplung von zentralen Diskussionen der scientific community und ein kaum zu rechtfertigender Schritt in einen Provenzialismus gerade für einen Sprachraum, der aufgrund seiner spezifischen Traditionen die internationale Debatte beleben könnte

Eberhard K. Seifert, Hamburg

\section{Fortsetzung von Seite 1}

Kommunistischen Manifest), lohnt es sich, das Problem in eines der Organisation sozialer Heterogenität umzuformulieren. Demgemäß dürfte man sich die Organisation einer starken ökologischen Konsumentenbewegung nicht als quasi mechanische Addition von gleichen Interessen vorstellen, sondern als die Schaffung eines hochdifferenzierten, aber nur „lose verkoppelten“ Interaktionsnetzes, das es Akteuren mit unterschiedlichen Präferenzen ermöglicht, gemeinsam stark zu werden.

Diese Position sah sich dem Vorwurf ausgesetzt, kollektives Handeln allzu statisch zu beschreiben und die Entstehung z.B. der neuen sozialen Bewegungen nicht erklären zu können - hierzu werden wir noch weitere Diskussionen organisieren. Auf der anderen Seite erwies sich dieser Ansatz als geeignet, die Schwächen und inneren Hemmnisse verschiedener Konsumenteninitiativen zu erklären. Die strategische Perspektive des heterogenen Bündnisses kam bereits in der Vielfalt der Tagungsbeiträge zum Ausdruck. Es wurde vorgeführt, wie eine Vielfalt von Konsumentenbewegungen an notwendigerweise ganz unterschiedlichen, sich aber tendenziell unterstützenden Punkten ansetzen: ökologische Beschaffungspolitik des Staates, Kundenberatung und selektive Einkaufspolitik des Handels, Aufbau einer Informations- und Beratungsstruktur für die Konsumenten, Konsumentenkampagnen gegen bestimmte Produkte und Hersteller, Hersteller-Verbraucher-Kooperation, ökologische Produktpolitik im Unternehmen.

Exemplarisch für diese Verknüpfungen seien einige Überlegungen zur erweiterten Mitbestimmung vorgestellt, mit denen auf der Tagung die Frage behandelt wurde; ob die neue gewerkschaftliche Forderung nach Produktmitbestimmung zu einem wirksamen Beitrag der Ökologiebewegung werden kann.

Die Forderung nach Produkt-Mitbestimmung beruht auf der Annahme, daß bei einer unmittelbaren Beteiligung der Produzenten diese als sozial verantwortliche Arbeiter und ökologisch sensibilisierte Privatpersonen die Entwicklung und Produktion schädlicher Produkte verhindern würden. In Bezug auf die ökologische Konversion bzw. Abschaffung von besonders schädlichen Produkten/Produktionsprozessen sind allerdings in den letzten Jahren sehr zwiespältige Erfahrungen gemacht worden: Auf der einen Seite hat das ökologische Bewußtsein und Verhalten der Arbeitnehmer im Privatleben, aber abgeschwächt auch im Betrieb, einen erheblichen Schub erhalten. Andererseits haben sich die Arbeitnehmer in Situationen, in denen das gesellschaftl. Intéresse an Schadensvermeidung sich gegen das privatwirtschaftliche Interesse des Einzelunternehmens durchzusetzen begann, mit dem Ziel der Arbeitsplatzsicherung hinter das ,gemeinsame Unternehmensinteresse“ gestellt häufig allein mangels Alternative.

An diesen Skandalen setzt die Forderung nach Produktmitbestimmung an: den zunehmenden, existenziellen Umweltproblemen und den Barrieren ihrer Vermeidung, der objektiven Ausschließung der Produzenten aus der Verantwor- 
tung und dem verbreiteten Desinteresse der Produzenten und ihrer Vereinnahmung für das Einzelunternehmensinteresse.

Die Forderung kommt also von oben, bezieht sich allerdings auf betriebl. Konversions-Initiativen, die sich seit 1981 insb. im Werften-Bereich und im beschäftigungsschwachen Norden entwickelt hatten. Diese Initiativen entstanden aus dem Anspruch nach „Rüstungskonversion“ und "alternativer Produktion" - das Konzept Mitbestimmung war eher eine nachrangige Zuordnung. In diesen Beschäftigungsinitiativen wurde versucht, vier gesellschaftl. Hauptprobleme zusammenzuführen und zu überwinden: die $\mathrm{Ar}$ beitslosigkeit, die brachliegenden Produktionskapazitäten, die Unter- bzw. Fälschversorgung der Region und die ungenutzten Fähigkeiten der Arbeitnehmer. Diese kleinen aktiven Gruppen von Beschäftigten haben, ohne Zuständigkeit und Zertifikat, alte Produktideen und neue Erfindungen aufgegriffen und Unternehmenslei tungen als Alternative zu Rüstungsproduktion und Entlassungen vorgehalten. Sie haben außerhalb der ausgetretenen Pfade gewerkschaftl. Programmatik und Vertretungspolitik mit Forschungs- und Entwicklungsabteilungen gestritten und mit Kommunalvertretern verhandelt

Doch die gewachsenen Interessensvertretungsstrukturen in den Betrieben lassen sich nicht bruchlos in eine solche Produktmitbestimmung erweitern, denn die Forderung der Gewerkschaften nach ökologischen Produkten war bisher maßgeblich als Mittel zum anderen Zweck, zur Sicherung bestehender und Schaffung neuer Arbeitsplätze, verstanden worden. Ökologie gilt als die Marktlücke, mit deren Hilfe der massive Arbeitsplatzabbau im industriellen Sektor kompensiert werden kann, als Motor eines qualitativen Wachstums. Wer diese Diversifikation einleitet und steuert, ist erst einmal zweitrangig; der Großteil der Gewerkschaften ist zufrieden, wenn die Unternehmer selbst aktiv werden

Zentraler Anknüpfungspunkt für die Betriebsräte ist der betriebliche Arbeits- und Gesundheitsschutz. Aufgrund der Mitbestimmungsrechte bei Regelungen über die Verhütung von Arbeitsunfällen und Berufskrankheiten sowie über den $\mathrm{Ge}$ sundheitsschutz, die Unterrichtungsrechte bei der Planung von Arbeitsverfahren und Arbeitsplätzen sowie der neuen Rechte aus der Gefahrstoffverordnung 86 haben die Betriebsräte hier zentrale Aufgaben und vielfältige Erfahrungen Es ist nun nach den Grenzen des daraus resultierenden Schutzselbstverständnisses $\mathrm{zu}$ fragen. Welche Arten von Umweltrisiken und -schäden werden darin erfaßt, welche nicht ? Ist das Schutzverständnis auf den eigenen Betrieb begrenzt oder weitet es sich unter bestimmten $\mathrm{Be}$ dingungen auf die Gesellschaft aus? Wie vermittelt sich das betriebsrätliche Schutzverhalten mit unternehmerischen Kosten- und Konfliktkalkülen ? Die bisherigen Konfliktverläufe haben gezeigt, daß die Betriebsräte, besonders wenn die Unternehmen unter massiven öffentlichen und ökonomischen Druck gerieten, gezwungen waren, das Interesse am Erhalt des Betriebs fast um jeden Preis (einschließlich den der Umwelt) zu vertreten. Gilt da für die Beschäftigten der Unternehmen etwas anderes?
Kaum, denn der normale industrielle Produktionsarbeiter ist i.d.R. von seinem Produkt, den Konsumenten des Produkts und evtl. Betroffenen getrennt. Die Gewährleistung seiner HauptArbeitsinteressen läuft nicht über das Produkt, sondern ist an die Prosperität seines Betriebes gebunden (Arbeitsplatz- und Einkommenssicherung, qualifizierte Tätigkeit). Das Interesse an (Konsum)Produkten ist über das Einkommen vermittelt: Je höher das Einkommen und je billiger die Produkte, desto höher ist das Konsumniveau.

Bei der Frage nach den unternehmensinternen Tätigkeiten der Produktmitbestimmung ist es sicher sinnvoll, die Differenzierung der Belegschaften, die Auswirkungen auf ihre Produktorientierung hat, zu berücksichtigen. So sind die Produzenten wesentl. an der Produktqualität und gesundheitsverträgl. Herstellungsverfahren interessiert, die Konstrukteure und Entwickler an einem funktionstüchtigen und verkaufbaren Produkt, die Verkäufer an den Kundenwünschen. Für eine wirksame Produktmitbestimmung scheint es unmöglich, dieses Spektrum an Fachwissen und produktbezogenen Interessenlagen in der Belegschaft zu mobilisieren und einzubeziehen. Nur diese Integration des betrieblichen Spektrums ist der Problemstellung angemessen und nicht eine Fortführung verkrusteter Vertretungsstrukturen.

Die Arbeitskreise "Alternative Produktion“ haben noch etwas anderes gelehrt: daß eine andere Produktpolitik nur bei einer starken externen Ergänzung möglich und sinnvoll ist. Die Kritik der industriellen Produktpalette der letzten Jahrzehnte ist entscheidend von außerhalb der Betriebe, d.h. von den neuen sozialen Bewegungen geleistet worden. Die betrieblichen Umstellungsinitiativen wären wohl ohne externe Stablilisierung (z.B. durch örtliche Vereine) alle binnen Kurzem eingegangen. Dabei waren diese Initiativen, die sich als Opposition gegen die traditionelle Interessenvertretungspolitik verstanden haben, noch gar nicht in die beiden Hauptzwänge hineingeraten, die bei einer Institutionalisierung der Produktmitbestimmung greifen würden: die enge Bindung an den betrieblichen Horizont und die Bindung an die Prosperität des Einzelbetriebs. Mitglieder des Betriebs werden nur kleine Ausschnitte der ökologischen Kreisläufe wahrnehmen, in die ihr Betrieb eingeschaltet ist. Die für sie unsichtbaren Teile der Kreisläufe müssen durch andere, externe Instanzen hinzugefügt werden. Und die Betrǐebsmitglieder sind über die Existenzbedingungen ihres Unternehmens erpreßbar - die Abhängikeit wird in dem Maße abnehmen, wie den internen Mitbestimmungsinstanzen externe Unterstützung zuwächst und regionale Alternativen geboten werden können.

Eckart Hildebrandt, Berlin

\section{Termine}

\section{Dez.-2.Jan}

Berlin: B.U.N.D.-Bundesjugendkongreß „Giftund Sondermüll"

19.-20.Jan

Arbeitskreis für Kooperation und Partizipation e.V. Berlin: Kooperatives Management Bestandsaufnahmen, Konflikte, Modelle, Zukunftsperspektiven

\section{0.-22.Jan}

Ev.Akademie Tutzing (in Nürnberg): Altlast Altzukunft

20.-22.Jan

Ev.Akademie Baden/VDI in Bad Herrenalb: „Hebt der Mensch das Weltklima aus den Angeln?"

30.Jan.-1.Febr

Ev.Akademie Loccum: Umnutzung von Industriebranchen - Initiativen entwickeln Konzepte

10.-12.Febr.

Ev.Akademie Loccum: Landwirtschaftlicher Strukturwandel in den 90er Jahren.

Entwicklungstendenzen, Wünsche, Gestaltungsspielräume (Loccumer Landwirtschaftstagung 1989)

13.-17. Febr.

Energie- und Umweltzentrum am Deister: Ökologisch orientierte Kommunalplanung

19.-24.Febr.

Energie- und Umweltzentrum am Deister: Ökologie und Pädagogik

10.-12. März

Ev.Akademie Tutzing: Der Wald steht schwarz und schweiget. Ein ökologisches Seminar mit Naturschützern und Forstleuten

10.-12.März

ISO: Kollektives Management „Was tun“ (clo M.Paulini, Bachstr. 22, 6100 Darmstadt)

3.-5. Mai

Ev.Akademie Tutzing: Ökologie und Ethik - ein interdisziplinärer Dialog

2.-4.Juni

Ev.Akademie Tutzing (auf Schloß NeunburgVormwald): Zum Beispiel: Solar - Wasserstoff Erneuerbare Energiequellen der Zukunft

26.-28.Juli

Taller Internacional Sobre Autogestion Latinoamericana in Mexico: „Replanteando las Formas Cooperativas para America Latina“ (Kontakt: G.Szell, Uni Osnabrück)

11.-15. Okt

Wiener Akademie für Zukunftsfragen: Ökologia 1989. Die Umweltmesse in Österreich. Der europäische Umweltkongreß

Impressum:
Herausgeber: Institut für ökologische Wirtschaftsforschung (IÖW) GmbH
Vereinigung für ökologische Wirtschaftsforschung (VÖW) e.V.
Redaktion: Dr. Klaus-Jürgen Scherer (V.i.S.d.P.), IÖW, Giesebrechtstraße 13,1000 Berlin 12, $030 / 8825281$
Konvertierung: Satzinform, Berlin 36 - Druck: Oktoberdruck, Berlin 36
Redaktionsschluß der nächsten Ausgabe: 15.2 .1989


(c) 20I0 Authors; licensee IÖW and oekom verlag. This is an article distributed under the terms of the Creative Commons Attribution Non-Commercial No Derivates License (http://creativecommons.org/licenses/by-nc-nd/3.o/), which permits unrestricted use, distribution, and reproduction in any medium, provided the original work is properly cited. 